REVUE DE L'INSTITUT

FRANÇAIS D'HISTOIRE

EN ALLEMAGNE

\section{Revue de l'IFHA}

Revue de l'Institut français d'histoire en Allemagne

$4 \mid 2012$

IFHA 4

\title{
Les « Mots de l'histoire », un laboratoire réflexif
}

\section{Christophe Duhamelle}

\section{OpenEdition}

\section{Journals}

Édition électronique

URL : http://journals.openedition.org/ifha/458

DOI : $10.4000 /$ ifha. 458

ISSN : 2198-8943

\section{Éditeur}

IFRA - Institut franco-allemand (sciences historiques et sociales)

\section{Édition imprimée}

Date de publication : 30 septembre 2012

Pagination : 176-186

ISSN : 2190-0078

\section{Référence électronique}

Christophe Duhamelle, «Les « Mots de l'histoire », un laboratoire réflexif », Revue de l'IFHA [En ligne],

4 | 2012, mis en ligne le 14 février 2013, consulté le 02 mai 2019. URL : http://

journals.openedition.org/ifha/458; DOI : 10.4000/ifha.458

Ce document a été généré automatiquement le 2 mai 2019.

(CIFHA 


\title{
Les « Mots de l'histoire », un laboratoire réflexif
}

\author{
Christophe Duhamelle
}

Huit « saisons » des Mots, de l'automne 2004 à juin 2012, cinquante-deux séances, centquarte intervenants - et une formule qui est restée très stable tout au long de cette période : un «duo", le plus souvent franco-allemand, et un commentaire. C'est pour ce dernier élément que l'évolution a été la plus nette : alors qu'au départ un invité extérieur était chargé de réagir aux deux premiers exposés, ce sont ensuite les organisateurs qui ont assuré à tour de rôle le commentaire en essayant de renouer, séance après séance, le fil directeur du séminaire.

Ce fil directeur était avant tout un projet dont l'article de Patrice Veit vient de retracer la genèse. C'était également une ambition, celle d'une sémantique historique que le jeu des décalages mais aussi des transferts permet, par une sorte de saut de côté réciproque entre France et Allemagne, de mieux apercevoir - le texte de Michael Werner revient plus en détail sur cet aspect. C'était en outre une manière de parler ensemble s'inscrivant dans un désir de travailler ensemble, le nœud, une fois par mois, de dilections, de coopérations, de curiosités et de réseaux que les huit saisons des « Mots » ont contribué à faire surgir - la contribution de Pierre Monnet détaille les linéaments de ce moment fédérateur.

Mais en dépit de ces solides permanences, le séminaire «Les Mots de l'histoire » s'est construit non selon un plan général enfanté dès le début, mais par une succession de choix. Chaque année, le petit groupe des organisateurs se réunissait, chacun tentant d'apporter une idée nouvelle, de remettre en mémoire les fulgurances non réalisées des années précédentes, de veiller à une juste répartition entre les thématiques ou les périodes. Restait alors à organiser concrètement les séances, et bien des idées séduisantes ont sombré corps et âme devant la difficulté de "trouver des intervenants » ou de les réunir à une date commune. Certaines sessions sont nées autour d'un mot, d'autres autour d'une question sur laquelle il fallait mettre des mots, d'autres encore autour d'un nom. Certaines ont mûri pendant trois ans, d'autres ont été improvisées en trois mois, quelques-unes - rares heureusement - ont dû être rafistolées en trois semaines. Et je crois 
pouvoir parler au nom de tous les commentateurs en disant que la découverte des textes, à un moment parfois désespérément proche du vendredi fatidique, a souvent chamboulé toutes les représentations que chacun avait pu se faire du sens de la séance.

Entre le cap bien tenu pendant ces huit années et ce caractère contingent de la programmation s'ouvre donc l'espace d'un bilan dont je tente ici, sans nourrir l'illusion d'une quelconque exhaustivité - et même en souhaitant conserver la dimension de surprise et parfois de désordre qu'a pu faire surgir, mois après mois, la fréquentation de ce rendez-vous savant et éclectique - de tracer quelques traits.

Cette présentation rétrospective ne prendra pas l'aspect d'un tableau d'honneur, même s'il est permis de rappeler qu'un séminaire qui a accueilli, entre beaucoup d'autres, Dominique Julia et Wolfgang Kaschuba, Barbara Stollberg-Rilinger et Roger Chartier, Peter Blickle, Jean-Claude Schmitt et Jan Assmann, ne peut pas avoir été entièrement mauvais. Tentons plutôt un regard global sur nos cent-quatre invités. Plus des trois quarts d'entre eux furent des hommes; ce n'est sans doute pas l'aspect le plus positif de la volonté qu'a eu le séminaire de refléter le plus fidèlement possible l'état de la recherche.

La liste des intervenants en revanche permet de souligner d'emblée une des caractéristiques du séminaire - Anna Karla en fournit à quelques pages d'ici la meilleure illustration - c'est-à-dire son souci de faire toute sa place à la jeune recherche. Pas seulement pour lui parler, mais aussi pour l'entendre. De ce point de vue, la «journée d'études junior » tenue en juin 2011 est emblématique d'une dimension qui s'est nourrie des configurations institutionnelles auxquelles «Les Mots» se sont adossés. Lors de cette journée en effet, ce sont avant tout les cursus bi-nationaux qui ont été à l'honneur. Au début, les doctorants de la Mission historique française en Allemagne qui sont intervenus plusieurs fois étaient davantage en vedette. Plus généralement, «Les Mots de l'histoire » ont veillé pendant huit saisons à ne pas être un séminaire de professeurs. Les programmes diffusés chaque année, joyeusement égalitaires en cela qu'ils n'indiquent pas les titres académiques, ne m'ont pas permis de tenter une solide étude statistique du statut universitaire des intervenants, mais nous nous souvenons de plusieurs séances qui ont fait dialoguer un jeune historien avec un collègue plus chevronné - l'option structurante du «duo franco-allemand » permettant de ce point de vue de transcender d'autres frontières statutaires, parfois plus lourdement pesantes.

Parmi tous les intervenants, nombreux furent ceux qui ne comprenaient pas la langue de l'autre. C'est un problème que les organisateurs se sont souvent posé et qui allait bien audelà d'une question purement pratique, celle de la compréhension réciproque. Fallait-il restreindre le dialogue franco-allemand sur «les mots de l'histoire » au cercle de ceux qui, dans leur recherche et leurs coopérations institutionnelles, le pratiquaient déjà courant ainsi le risque d'un séminaire qui aurait pu s'intituler «Les mots de l'entre-soi franco-allemand»? Fallait-il au contraire provoquer ce dialogue, confronter celles et ceux qui découvriraient véritablement les mots de l'autre - au risque cette fois-ci d'aboutir, littéralement, à un dialogue de sourds. En vision cavalière, le programme a effectué un compromis entre les deux options et nous avons souvent défié la fameuse «barrière de la langue " puisque notre postulat était que cette barrière pouvait se transformer en un observatoire particulièrement éclairant - le commentaire ayant alors la fonction d'interprétariat linguistique autant que d'interprétation réflexive. Rétrospectivement, il semble d'ailleurs que le danger n'était pas aussi grand que nous pouvions le craindre. Chacun se souviendra de séances où la traduction improvisée a jailli 
de tous côtés et, finalement, a toujours permis de s'entendre. Les mots ont dans ce séminaire toujours constitué une problématique davantage qu'un problème.

Une problématique, mais autour de quels thèmes? Le bilan des 52 séances peut être appréhendé selon plusieurs lignes de partage.

La première serait de les répartir en fonction des grandes césures classiques des sousspécialités historiennes. De ce point de vue, les «Mots» ont bien été le reflet des tendances historiographiques des dernières années. L'histoire culturelle a été davantage représentée que l'histoire économique - et aucune séance ne s'est interrogée sur un débat longtemps essentiel, certes selon des modalités diverses, pour les historiens des deux pays : la relation entre les mots et les chiffres, les catégories et les agrégats, les mots des sources et les termes de la statistique. Un retour vers la tradition braudélienne ou vers les apports de l'École de Bielefeld fait ainsi apparaître d'autant plus révélatrice l'absence d'une séance confrontant «Structure » et «Struktur ». En y regardant de plus près, même les rares séances "économiques » ont reflété l'évolution du champ, consacrées qu'elles étaient au «travail » et à la " consommation » - mais jamais à la production, sans même parler, pour rassembler les deux remarques, des structures de production, ou d'un terme qui a pourtant ses lettres de noblesse dans la circulation des mots entre allemand et français: le prolétariat. De même, pour autant qu'il soit aisé ou même opportun de distinguer des séances d'histoire sociale, celles-ci se sont davantage centrées sur les formes concrètes de la communauté (la commune, la maisonnée) que sur celles de la confrontation.

En soulignant la prédominance d'une histoire «culturelle » il ne s'agit pourtant pas de renvoyer à la définition étroite du terme, mais bien à l'évolution globale, et touchant les deux pays, vers une histoire moins soucieuse d'établir un sens surplombant de l'Histoire que d'explorer les façons dont les acteurs de l'histoire construisaient leur propre expérience. Ainsi, les séances sur le paysage ou la région ont-elles illustré la manière dont une attention renouvelée envers l'espace est liée à la notion de construction sociale des horizons, des appartenances et des réseaux. C'est d'ailleurs en cela, profondément, que l'une des ambitions majeures du séminaire était bien de son époque historiographique : celle de marier constamment l'interrogation sur les mots des historiens et sur les mots des acteurs ou des sources. La réflexivité binationale, ou plutôt bilingue, rejoint ici intimement l'attention aiguë portée au bilinguisme de l'historien entre compréhension et explication. De manière révélatrice, l'un des lieux historiographiques les plus féconds pour cette réflexion, c'est-à-dire le travail sur l'écriture de soi et le témoignage, a fourni à lui seul trois de nos 52 séances. Communication, image, rituel, émotions : au travers des sessions " politiques ", « sociales » ou autres, court ainsi une thématique cachée qui peutêtre s'est exprimée en 2008 dans la journée d'études sur la «Lebenswelt» dont, si je me souviens bien, nous n'avons jamais réussi à donner une traduction satisfaisante.

Une seconde ligne de partage semble apparaitre en considérant la liste de nos rendezvous mensuels : celle qui isolerait la quinzaine de séances explicitement consacrées à l'historiographie. Certaines d'entre elles interrogèrent l'articulation entre la discipline historienne et d'autres spécialités soucieuses de l'historicité (l'histoire du droit, l'ethnologie) ou encore la constitution de «champs » internes à la discipline (l'histoire universelle, l'histoire de l'environnement). D'autres, sans doute trop peu nombreuses, furent consacrées à la périodisation, au découpage chronologique - mais leur rareté est sans aucun doute l'indice d'une conjoncture historiographique pour laquelle l'ordonnancement de l'évolution cesse d'être la priorité. Un troisième type de séances 
pourrait entrer dans cette catégorie « historiographique » : celles consacrées à de grands concepts problématiques, de la mémoire aux représentations, de l'individualisation au discours.

Mais à peine a-t-on posé cette ligne de partage qu'il faut à nouveau la brouiller. Car tout cela fut présent également dans les autres séances. La configuration différente des répartitions par champs ou par disciplines d'un côté du Rhin et de l'autre est, par exemple, apparue de façon particulièrement marquée dans une séance apparemment fort innocente de ce point de vue, celle sur "pèlerinage/Wallfahrt». Et certains "grands paradigmes » ont été les invités quasi permanents du séminaire alors qu'aucune séance ne leur a été spécifiquement consacrée. Je pense en particulier à la notion de "Moderne ", si souvent réapparue.

Les séances les plus explicitement "historiographiques» ne sont donc pas nécessairement celles où ont le plus vivement surgi les interrogations sur ce que veut dire "une historiographie». C'est sans doute ici que l'imprévu des rencontres et le vagabondage des débats - dont la richesse et la capacité à s'embarquer vers des rives que le titre du jour ne permettait pas d'apercevoir resteront, j'en suis sûr, la part la plus fervente de la mémoire des « Mots » - rejoignirent le mieux une des idées directrices du séminaire. Cette idée est que les manières de dire sont toujours des manières de faire, mais que bien souvent elles les résument, elles les présupposent, elles les informent en leur donnant, par un langage commun, la force de ce qui va de soi. La langue des historiens aussi est faite de conventions qui gardent leur évidence jusqu'à ce qu'elles croisent l'énigme d'une convention contraire. Le séminaire « Les Mots de l'histoire » doit une partie de sa richesse à ce parallèle constant et fécond entre un discours réflexif sur les concepts reconnus comme tels, situés dans leur généalogie et leur portée, et le heurt surprenant entre des conventions de parole révélant la structuration du champ ou les présupposés de la socialisation scientifique. En ce sens, le séminaire a renvoyé autant à une sociologie comparée des historiens allemands et français qu'à leur usage raisonné du vocabulaire. Les "Mots de l'histoire " auraient été plus pauvres s'ils n'avaient été que « les concepts de l'historien ». Car il ne s'est pas agi seulement de la puissance, du sens, de l'utilité opératoire des concepts - il s'est agi également de leur statut, c'est-à-dire de leur valeur sociale dans ce monde social qu'est celui des historiens.

Une troisième ligne de partage sera-t-elle moins malhabile à ordonner le bilan du séminaire? Elle se focaliserait davantage sur les « couples de mots " eux-mêmes, ceux que par commodité j'ai jusqu'ici parfois réduits au vocable français alors que ces faux jumeaux forment pour ainsi dire la marque de fabrique des intitulés du séminaire, c'est-àdire l'attelage entre un mot allemand et un mot français le plus souvent, parfois deux ou trois d'un côté ou de l'autre. Cet étendard du séminaire n'est pourtant pas apparu tout de suite : lors de la première saison, des formulations différentes étaient encore présentes. Mais le « couple» a vite pris le dessus, non comme une facilité, mais plutôt comme une gageure. C'est en effet à travers lui que nous avons acquis l'habitude de poser les problèmes. Car ces « couples » ne se sont jamais imposés comme des évidences. J'en veux pour seule preuve les quelques termes qui reviennent dans plusieurs séances, mais associés, ou opposés, à des termes différents : caméralisme/Kameralismus sont là en 2005-2006 et en 2009-2010, mais la première fois le caméralisme est en regard de la physiocratie, suggérant deux modalités concomitantes mais distinguées de l'économie politique ; la seconde fois, il est associé dans les deux langues à "sciences camérales", faisant cette fois porter l'accent sur la circulation européenne des savoirs d'État. Identité 
et Identität reviennent deux fois également, dans des sessions très différentes, l'une portant sur les conceptions différentes de la citoyenneté, l'autre sur le couple religion/ confession. La même remarque vaudrait pour les deux occurrences de Selbstzeugnisse dans des titres de séances. C'est assez dire qu'entrechoquer les mots n'a jamais eu pour seul but de procéder à une juxtaposition muséale et de cultiver un plaisir d'entomologiste polyglotte, mais qu'il s'est toujours agi de les heurter l'un à l'autre en essayant de choisir un angle qui ferait jaillir l'étincelle.

C'est qu'il y a eu bien des façons d'agencer nos « couples de mots». Parfois, l'apparente similarité a été mise en exergue : Region/Région, Absolutisme/Absolutismus - et même ce cas limite auquel nous avons finalement renoncé: gender/gender, qui finalement a sagement bifurqué vers Geschlecht, gender/Genre, gender. Tout le charme de ces appariements était de découvrir, par contraste, les racines différentes aboutissant à ce rameau apparemment commun. Dans d'autres cas, au contraire, la différence des deux termes a été posée de façon presque brutale, peut-être énigmatique pour les amis du séminaire découvrant le programme : Physiocratie/Kameralismus, dont j'ai déjà parlé, ou encore Écritures de soi/Selbstzeugnisse. Le couple des mots signalait alors d'emblée que ce n'était pas de la même chose que l'on parlait, ou que l'on n'avait pas choisi les même termes pour en parler - mais l'horizon d'attente de la séance était de provoquer un sursaut réflexif permettant de dégager tout ce que cette différence voulait dire. Ce funambulisme à deux fils connut un cas presque limite, qu'il fallut faire prudemment précéder de la mention "Deux intraduisibles » : la séance intitulée "Staatskirchenrecht/ Laïcité »

Mais, là encore, les types de couples ne permettent pas d'établir une typologie des séances. Car derrière l'apparente gémellité ou le contraste affiché, le choix d'un seul terme dans chacune des langues ou au contraire d'une "nébuleuse » de notions, s'est toujours profilé un ressort récurrent du séminaire, celui de la traduction - un ressort, et non un thème. Il nous est certes bien arrivé de parler de la langue, de ses contraintes, de ses possibilités, des étymologies - du mot lui-même. Mais nous nous sommes plus souvent attachés à le replacer dans les configurations, les familiarités et les contrastes dans lesquels il s'inscrivait. De la traduction, nous sommes passés à la tradition, à la culture scientifique qui ancre un terme dans une constellation de significations. Les différents types de couples nous ont par conséquent tous permis de faire porter l'interrogation sur l'au-delà du mot, sur le contexte historique et historien que, tel les fleurs de papier japonais chères à Marcel Proust, le mot se déploie dans toutes ses irisations dès qu'il est plongé dans le bol d'eau qu'était, pour nous, l'épreuve de la traduction. La proximité des deux mots fait d'autant mieux voir la dissemblance de leurs arrière-plans; leur contraste oblige au contraire à considérer la parenté de ce qu'ils cherchent différemment à dire.

Il est par conséquent révélateur que nous n'ayons jamais dédié de séance à la traduction elle-même, comme activité d'écriture que pourtant la plupart des participants au séminaire pratiquaient, soit pour traduire et citer les sources, soit pour prendre la parole dans l'autre langue, soit pour exercer une activité de « passeur » des travaux des autres. Il aurait été concevable, par exemple, de consacrer un vendredi matin à un problème bien réel et moins trivial qu'il n'y parait: l'historien français doit éviter les répétitions, l'historien allemand ne peut remplacer constamment un Begriff par un autre, et le traducteur se demande ce qu'il doit faire. En parcourant mes notes sur les débats qui ont eu lieu à l'occasion du séminaire, je m'aperçois que nous serions bien en peine de proposer une sorte de « dictionnaire bilingue des Mots de l'histoire ». 
Les mots nous ont un peu servi de prétexte pour du contexte. Mais pas n'importe quel prétexte puisque c'est précisément de cette disjonction que nous avons joué. C'est dans l'embarras du langage, révélé par le mouvement d'une langue à l'autre, qu'on peut apercevoir à quel point ce que l'on dit est infiniment plus que ce que l'on croit dire.

Il est donc plus malaisé qu'il y paraît d'établir un bilan thématique des "Mots de l'histoire » car les répartitions que suggère une simple lecture des programmes sont contredites par le souvenir des séances, de leur variété, des chemins de traverse qu'elles empruntaient souvent. L'inconfort ressenti au moment du bilan est toutefois l'indice d'une réussite de ces huit années. S'il est si difficile d'établir une typologie des séances des « Mots de l'histoire », c'est bien parce que l'unité du séminaire est profonde et qu'audelà des spécificités de chaque session s'est toujours profilé un horizon commun. Les «Mots » nous ont servi de sonde pour mesurer les sédimentations qui forment le socle à la fois des objets que nous étudions et de nos manières de les étudier. En géologie, ce sont les failles et les plissements qui font affleurer ces sédimentations. Nos failles et nos plissements ont été nos couples de mots et, à travers eux, ce que révèle de soi la différence de l'autre. C'est en cela que la dimension franco-allemande du séminaire prend tout son sens. Il y entre bien sûr la volonté d'apporter une information réciproque sur les deux historiographies, ainsi que sur les deux histoires, et sur leurs interrelations. Mais constater les différences n'aurait que peu d'intérêt. S'accorder sur un plus petit dénominateur commun n'en aurait guère davantage. C'est le retour réflexif sur soi qui fait au fond tout le prix de l'opération. Les meilleures séances des "Mots de l'histoire " sont donc celles où, quel que soit le thème abordé, chacun a pu se sentir concerné par les questions qui venaient au jour.

C'est pourtant, pour finir, ce cadre franco-allemand que je souhaite interroger car il fut souvent mis en question, y compris au sein du cercle des organisateurs, et le projet d'élargir les «Mots » vers un horizon plus international fut fréquemment caressé. Une des journées d'études clôturant les saisons du séminaire a été consacrée à la pertinence de l'échelle européenne et certaines séances abordèrent le thème de l'histoire universelle comme celui de la globalisation. Il est en outre apparu à de nombreuses reprises - pas seulement à propos du gender - que les historiens français et allemands puisent souvent une partie de leur bagage disciplinaire et de leur vocabulaire à d'autres sources. Les parcours des jeunes chercheurs sont un indice parmi d'autres d'une internationalisation de la recherche qui peut faire apparaitre un séminaire franco-allemand comme la continuation, même sous une forme duale, d'une conception nationale dépassée de la tradition scientifique. Pour ce qui est des " Mots ", on ne peut écarter d'un revers de main l'idée qu'une langue scientifique commune - le fait qu'elle soit l'anglais, et donc pas la nôtre, pourrait ici céder le pas à l'avantage qu'elle aurait d'être universellement pratiquée - résoudrait un nombre considérable de problèmes. D'un autre côté, rappeler $a$ contrario que la diversité des approches, incarnée entre autres dans la diversité des langages, constitue incontestablement une richesse et une incitation à la réflexivité rappeler également que l'apparente simplification d'une langue universelle aurait comme redoutable corollaire d'établir la fausse évidence d'une transparence du langage - ne justifie pas pour autant que cette pluralité, saisie au travers des mots et de tout ce que leur usage véhicule, soit réduite au pas de deux du français et de l'allemand.

Le cadre franco-allemand ne peut donc se justifier entièrement par lui-même. Un historien ne peut certes pas oublier qu'il s'orne d'une valeur singulière, celle de symbole et de moteur européen. Mais le passage du Rhin est trop étroit pour étreindre à lui seul 
l'horizon de la recherche. Ce n'est donc pas dans le paradoxe étriqué d'une internationalisation limitée à deux qu'il trouve son sens, mais comme laboratoire de la réflexivité.

Pour faire levier, il faut deux points, pas un seul point, mais pas quinze points. 52 séances des « Mots de l'histoire » - et chacun saurait pourtant dire son deuil des séances qui n'ont pas eu lieu. Le séminaire pourrait être sans fin et il faut pourtant bien qu'il se termine. 52 séances dont le fil conducteur franco-allemand seul a permis que chacun assiste à des débats totalement éloignés de sa "spécialité » et puisse, malgré cet éloignement ou grâce à lui, en tirer profit. Le couple de mots testé à chaque session, résistant, stimulant, est plus que la "marque de fabrique" du séminaire. Il est aussi la bonne échelle de réflexivité, celle qui lui permet de s'approfondir et de fournir ensuite les moyens d'être appliquée à une ouverture internationale plus large. À être abordée d'emblée comme horizon naturel, l'internationalisation présente en effet le danger d'aboutir paradoxalement à une fragmentation: les grands colloques internationaux se déclinent par exemple en sessions compartimentées où se retrouvent toujours les mêmes spécialistes, "hors sol», si l'on ose dire. Par contraste, la spécificité des «Mots de l'histoire » réside en définitive moins dans leur dimension franco-allemande que dans ce qui s'avère de plus en plus rare : leur refus de la spécialisation, soit sur une période, soit sur une thématique, soit même sur une discipline. Tendu entre deux mots, unifié par son cadre binational, le séminaire a permis d'explorer la complexité de ce qui inscrit tout historien dans une multiplicité de contextes, touchant à la sociologie de la science autant qu'à l'apprentissage du langage disciplinaire, et il a proposé comme horizon commun non une évidence universelle partagée, mais l'exigence universellement partagée du refus de l'évidence. Il faut une certaine intensité de la démarche pour permettre à cette exigence de déborder son champ de spécialisation, fût-il ouvert au monde, et c'est cette fonction que peut continuer à remplir un séminaire franco-allemand. Les deux mots de chaque séance formaient les deux points d'un levier d'évidence.

En ce sens, la difficulté qu'il y a à établir un bilan thématique des huit années de « Mots de l'histoire » n'est pas seulement l'indice de la cohérence établie par son fil directeur. Elle fonde également la justification même du séminaire - un laboratoire réflexif qui a pris le temps, sur les sujets les plus divers, d'effectuer obstinément un sondage en profondeur de l'historicité du langage scientifique.

\section{AUTEUR}

\section{CHRISTOPHE DUHAMELLE}

Christophe Duhamelle est directeur du Centre de Recherches Historiques (UMR 8558) à l'École des Hautes Études en Sciences Sociales. 